\title{
Kernos
}

Revue internationale et pluridisciplinaire de religion grecque antique

4 | 1991

Varia

Pausanias et les origines mythiques de Delphes : éponymes, généalogies et spéculations étymologiques

\section{Léon Lacroix}

\section{Q OpenEdition}

\section{Journals}

Édition électronique

URL : http://journals.openedition.org/kernos/308

DOI : $10.4000 /$ kernos.308

ISSN : 2034-7871

\section{Éditeur}

Centre international d'étude de la religion grecque antique

\section{Édition imprimée}

Date de publication : 1 janvier 1991

Pagination : 265-276

ISSN : 0776-3824

\section{Référence électronique}

Léon Lacroix, «Pausanias et les origines mythiques de Delphes : éponymes, généalogies et spéculations étymologiques », Kernos [En ligne], 4 | 1991, mis en ligne le 11 mars 2011, consulté le 01 mai 2019. URL : http://journals.openedition.org/kernos/308 ; DOI : 10.4000/kernos.308 
Kernos, 4 (1991), p. 265-276.

\section{PAUSANIAS ET LES ORIGINES MY'THIQUES DE DELPHES : ÉPONYMES, GÉNÉALOGIES ET SPÉCULATIONS ÉTYMOLOGIQUES}

Dans son livre Pausanias à Delphes, Georges Daux a reproduit le texte de la périégèse delphique, qu'il a traduit et commenté. Mais il a laissé de côté la longue introduction qui précède la description du sanctuaire, ainsi que certains passages sur lesquels la fouille ne peut rien nous apprendre (voir p. 2 de l'introduction). C'est le point de vue légitime de l'archéologue qui veut confronter les renseignements du Périégète avec les données de la fouille. Le point de vue est évidemment différent si l'on s'attache à l'œuvre elle-même de notre auteur, à la manière dont il a conçu sa tâche et dont il l'a réalisée. Dans ce cas, on peut dire que les $\lambda$ ó yor n'ont pas moins d'importance que les $\theta \varepsilon \omega \rho \eta \dot{\mu} \mu \alpha \alpha^{1}$. Ils font partie d'un ensemble et l'on ne saurait les négliger. Certains d'entre eux concernent les origines mythiques de Delphes. J'ai voulu les examiner pour en déterminer l'intérêt et la signification, mais en m'en tenant bien entendu au témoignage de Pausanias ${ }^{2}$.

En abordant son sujet, le Périégète fait observer qu'il existe des traditions abondantes et variées au sujet de Delphes et qu'il y en a plus

1 Voir Chr. HABICHT, Pausanias und seine "Beschreibung Griechenlands", Munich, 1985 , p. 32 ; sur cette question, voir aussi les observations de Fr. Chamoux, Pausanias historien, in Mélanges André Tuilier, 1988, p. 42. On a depuis longtemps fait observer que l'œuvre de PAUSANIAS est tout autre chose qu'un guide du voyageur, tel que nous pourrions le concevoir. Voir $R$. Heberdey, Die Reisen des Pausanias in Griechenland, Abh. des archäolog.epigraph. Seminares der Univ. Wien, X (1894), p. 2 : "Gegenstand der

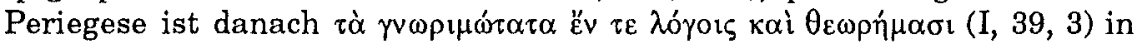
ganz Griechenland $(1,26,4)$, d. h. eine Auswahl dessen, was an Überlieferungen ( $\left.\lambda \circ \gamma_{\gamma \circ \imath}\right)$ und Sehenswürdigkeiten aller Art $(\theta \varepsilon \omega \rho \eta ́ r \mu \alpha \tau)$ dem Verfasser belangvoll erschien".

2 Pour une mise au point des questions relatives à l'oracle de Delphes et un recueil des principaux textes, voir P. AMANDRY, La mantique apollinienne à Delphes, Essai sur le fonctionnement de l'oracle, Paris, 1950 (cité ici P. AMANDRY, Mantique). Je renverrai aussi au livre de G. Roux, Delphes, son oracle et ses dieux, Paris, 1976. Les textes grecs sont cités d'après l'édition de PaUsanias de M.H. Rocha-Pereira (coll. Teubner). 
encore au sujet de l'oracle d'Apollon ${ }^{3}$. Dans son introduction, il traitera successivement de l'oracle, du temple d'Apollon, de la ville de Delphes, des concours pythiques et de l'Amphictyonie (X, 5, 5-X, 8, 5). Avant de passer à la ville et à ses origines, je résumerai les informations de Pausanias sur l'oracle et sur le temple. Nous apprenons ainsi qu'aux temps les plus anciens ( $\tau \dot{\alpha} \alpha \dot{\alpha} \rho \chi \alpha$ เó $\alpha \tau \alpha)$ ), l'oracle appartenait à Gé ou, d'après un poème attribué à Musée (2 F 11 Diels-Kranz ${ }^{6}$ ), qu'il aurait été

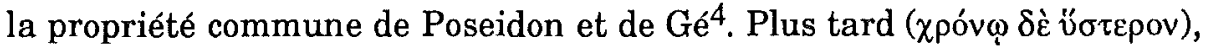
Gé céda sa part à Thémis, laquelle en fit cadeau à Apollon. Quant à Poseidon, il procéda à un échange qui lui permit d'obtenir Calaurie, en face de Trézène ${ }^{5}$. L'histoire du temple commence aussi aux temps les

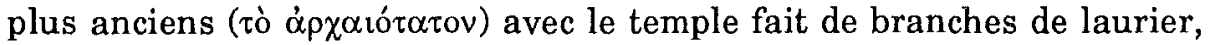
auquel succéda le temple construit par des abeilles à l'aide de cire et de plumes, qui fut envoyé par Apollon chez les Hyperboréens ${ }^{6}$, puis vint le temple en bronze, englouti, disait-on, dans une crevasse, où il fondit sous l'action du feu. Le quatrième temple, cuvre de Trophonios et Agamédès, était un édifice en pierre, qui fut détruit par le feu la première année de la 58e Olympiade $(548 / 547)^{7}$. Puis Pausanias passe directement à l'édifice qui subsistait de son temps, construit aux frais des Amphictyons par un architecte corinthien, Spintharos 8 .

Nous arrivons à la ville de Delphes $(\mathrm{X}, 6)$ et Pausanias, ici encore, va s'occuper essentiellement des origines. La ville la plus ancienne aurait été fondée à cet endroit par Parnassos ${ }^{9}$. Fils de la nymphe Kléodôra, Parnassos bénéficiait, comme d'autre héros, d'une double paternité : celle d'un dieu, Poseidon, et celle d'un homme, Kléopompos. Il donna son nom à la montagne, le Parnasse, ainsi qu'à un vallon

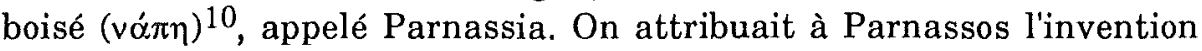

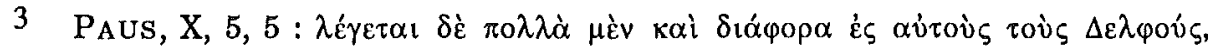

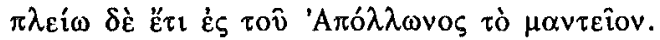

4 Textes cités et traduits par P. AMANDRY, Mantique, p. 127, n LXI; p. 60, n LXII.

5 Sur cet échange, voir aussi PAus., II, 33, 2 ; cf. G. Roux, Delphes, p. 29.

6 Sur ce transfert miraculeux, voir mon article dans Bull. Acad. royale de Belgique. Classe des Lettres (1983), p. 93.

7 C'était en fait le premier édifice qui n'appartenait pas aux temps mythiques : G. Roux, Delphes, p. 37.

8 PaUsanias a donc omis le temple dit "des Alcméonides», construit dans le dernier quart du VIe siècle; voir à ce sujet les remarques de P. AMANDry, Bull. Acad. royale de Belgique, Classe des Lettres (1989), p. 26.

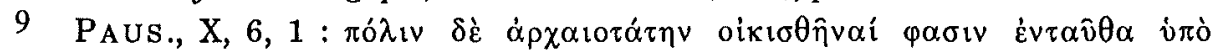

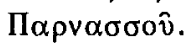

10 Nór $\pi$ est cité par le scholiaste de PIndare, Pyth., I, inscr. B parmi les *anciens» 
de l'ornithomancie. La ville qu'il avait fondée fut engloutie par le déluge survenu au temps de Deucalion. Parmi les habitants, ceux qui avaient pu échapper au cataclysme, guidés par les hurlements des loups

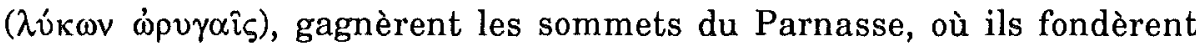
une ville qu'en raison des circonstances, ils appelèrent Lykôreia ${ }^{11}$. Selon une autre tradition, Lykôreia devrait son nom à Lykôros, fils d'Apollon et de la nymphe Kôrykia. Nous arrivons ainsi à une généalogie de Delphos, l'éponyme de Delphes. Lykôros eut pour fils Hyamos, qui fut le père de Kélainô. D'Apollon et de Kélainô naquit Delphos. Il existait bien entendu des variantes. L'une d'entre elles faisait intervenir un autochtone $(X, 6,4)$ du nom de Kastalios ${ }^{12}$. Il eut pour fille Thyia, qui fut la première prêtresse de Dionysos et qui donna son nom aux Thyiades. Dans cette généalogie, Delphos est le fils d'Apollon et de Thyia. Dans une autre, il a pour mère Mélaina, fille de Képhisos.

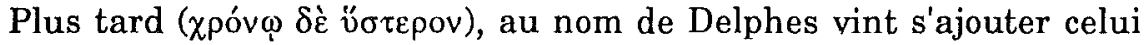
de Pythô, connu d'Homère, et Pausanias renvoie son lecteur au Catalogue des vaisseaux (Il., II, 519). Des érudits qui voulaient tout expliquer par des généalogies ${ }^{13}$, attribuaient à Delphos un fils appelé Pythès; c'est sous le règne de ce dernier que Delphes aurait pris le nom de Pythô. Toutefois, la tradition la plus répandue ${ }^{14}$ mettait Pythô en rapport avec $\pi v ́ \theta \varepsilon \sigma \theta \alpha \imath$, "pourrir». La légende voulait qu'un monstre tué par les flèches d'Apollon ait pourri à cet endroit. Selon les poètes, dont Pausanias invoque le témoignage, ce monstre était un dragon qui veillait sur l'oracle de Gé. Les considérations qui suivent concernent les actes d'impiété commis par ceux qui pillèrent le sanctuaire, en commençant par Krios, fils d'un tyran eubéen $(X, 6,6)$, pour aboutir à Néron, qui déroba à Delphes cinq cents statues $(X, 7,1)$.

Avec Pausanias et son goût bien connu pour le passé ${ }^{15}$, nous remontons toujours aux temps les plus anciens, ce qui ne peut se faire sans recourir à la mythologie. Cette tendance s'affirme tout au long de l'exposé consacré à l'oracle, au temple d'Apollon et à la ville de Delphes. Elle se manifeste aussi dans la suite de cette introduction,

noms de Delphes; cf. L. Lerat, Krisa, in Mélanges Charles Picard, II (1949), p. 627, n. 4 .

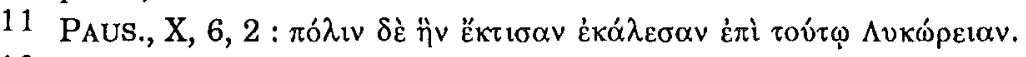

12 Ailleurs (PAUS., VII, 18, 9; schol. EUR., Or., 1094), Kastalios est présenté comme le fils de Delphos, ce qui montre bien le caractère artificiel de ces généalogies; sur Kastalios, doublet de Kastalia, voir PAUS., X, 8, 9.

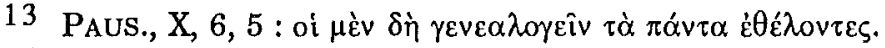

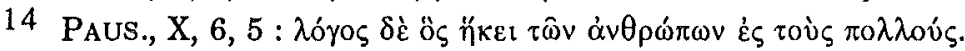

15 Chr. НАBICHT, op. cit., p. 34-35. 
quand on en arrive à l'institution des concours pythiques $(\mathrm{X}, 7,2)$ et aux origines de l'Amphictyonie, qui devait son nom à Amphictyon, fils de Deucalion $(\mathrm{X}, 8,1)$.

Dans cette histoire mythique, Pausanias suit un ordre chronologique. La ville la plus ancienne, celle de Parnassos, se situe avant le déluge. Après le déluge, nous passons à la fondation de Lykôreia, pour aboutir enfin à celle de Delphes. La mention de Pythô après Delphes constitue un sérieux accroc à l'ordre chronologique, car le nom de Pythô est bien attesté dans les poèmes homériques (Il., II, 519; IX, 405). Pausanias a recueilli la tradition qui fait de Pythès le fils de Delphos, mais il n'a pu s'empêcher de noter la confiance excessive que certains érudits accordaient aux généalogies ${ }^{16}$. Sans doute préférait-il l'antique légende de fondation, qui racontait la victoire d'Apollon sur le dragon et qui associait le nom de Pythô à $\pi u ́ \theta \varepsilon \sigma \theta \alpha$, «pourrir».

Une autre remarque vient naturellement à l'esprit. Il suffit de parcourir l'œuvre de Pausanias pour y découvrir quantité d'éponymes qui sont censés avoir donné leur nom à des peuples, à des villes, à des montagnes, à des fleuves et à des sources. Dans la partie de l'introduction consacrée aux origines de Delphes, les éponymes sont particulièrement nombreux. Nous voyons ainsi se dessiner un véritable paysage avec le mont Parnasse (Parnassos), l'antre corycien (Kôrykia), la source Castalie (Kastalios) et le fleuve principal de la Phocide, le Céphise (Képhisos). Des villes prennent place également dans ce paysage avec Lykôros, Delphos et Pythès.

Les éponymes sont généralement pourvus de généalogies qui les associent aux traditions légendaires de la cité ou de la région ${ }^{17}$. À Delphes, certains d'entre eux sont ainsi rattachés aux grandes divinités du sanctuaire. Fils de Poseidon, Parnassos est associé à la génération divine la plus ancienne. Apollon apparaît ensuite comme ancêtre de Delphos. Dionysos n'est pas oublié. Dans une des généalogies mentionnées par Pausanias, il est question de Thyia : première prêtresse du dieu, elle aurait institué en son honneur des cérémonies religieuses (ő $p(\alpha)$ et donné son nom aux Thyiades ${ }^{18}$. Delphos est privilégié, car il est doté de plusieurs généalogies ${ }^{19}$. Quant à Pythès, fils de Delphos, son

16 Voir ci-dessus, n. 13.

17 Au sujet des généalogies des héros, on notera l'observation de PAUSANIAS, I, 38, 7 , sur la diversité des traditions quand elles ne trouvent pas un appui dans le témoignage des poètes; sur cette diversité, voir aussi VIII, 53, 5 .

18 Sur les Thyiades, voir G. Roux, Delphes, p. 178 sq.

19 Sur les généalogies de Delphos, voir mes Etudes d'archéologie numismatique, 
existence est le résultat d'une élaboration érudite et l'on reconnaîtra en lui une création tardive.

Mais il n'en est pas de même de tous les éponymes. Hellanicos

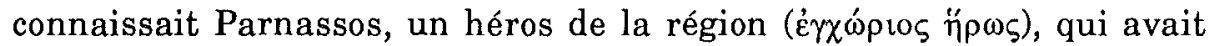
donné son nom au Parnasse ${ }^{20}$. Les renseignements qu'Hérodote (VII, 178) nous a conservés au sujet de Thyia montrent qu'il ne s'agit pas de l'invention de quelque ingénieux mythographe, mais d'une divinité qui avait son sanctuaire dans les environs de Delphes et dont le culte était associé à celui des Vents ${ }^{21}$. Quant à Delphos, il est cité par Eschyle dans le prologue des Euménides : lorsque Phoibos, succédant à Gaia, à Thémis et à Phoibé, gagne la région du Parnasse, il reçoit l'hommage du peuple «et de son roi, Delphos, pilote du pays» ${ }^{22}$. En partant de ce texte, André Plassart a pu proposer de reconnaître Delphos au fronton du temple archaïque de Delphes ${ }^{23}$.

Les spéculations des anciens sur les origines des peuples et des villes font aussi une place aux étymologies. Il faut entendre par là des approximations fondées sur de simples "rapprochements paronomastiques", pour reprendre l'expression de Clermont-Ganneau ${ }^{24}$. Le nom de Pythô avait donné naissance à une de ces "étymologies", directement liée à l'installation du dieu dans son sanctuaire. Selon la tradition rapportée dans la Suite Pythique, Apollon s'assura la possession de l'oracle en triomphant d'un monstre qu'il perça de ses flèches. Après sa victoire, le dieu condamna le monstre à "pourrir» ( $\pi \dot{v} \theta \varepsilon v$, v.

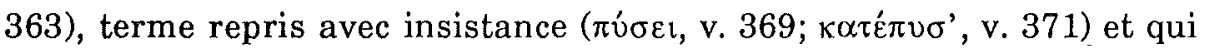
justifie le nom de Pythô, ainsi que l'épiclèse Pythios ${ }^{25}$. L'explication est reprise par Pausanias $(\mathrm{X}, 6,5)$ qui, attentif à l'évolution de la langue,

Paris, 1974, p. 38.

204 F 196 Jacoby (schol. Apoll. RHOD., II, 711) : ' $\Omega v o \mu \alpha ́ \alpha \sigma \eta \eta$

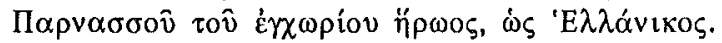

21 Sur le culte des Vents et l'emplacement du sanctuaire, voir G. Roux, Delphes, p. 200-201. Selon la tradition, Thyia est fille de Képhisos (HDT.) ou de Kastalios (PAUS.).

22 Esch., Eumén., 16 (trad. P. Mazon). Sur le prologue des Euménides et la succession des généalogies divines, voir les observations de P. AMANDRY, Mantique, p. 201, n. 2 et p. 214, n. 2.

23 A. Plassart, in $R E A, 42$ (1940), p. 298. Sur cette interprétation, voir Erika SIMON, LIMC, III (1986), s.v. Delphos.

24 J'ai cité plusieurs exemples de ces «rapprochements paronomastiques» in Bull. Acad. royale de Belgique, Classe des Lettres (1989), p. 93 sq.

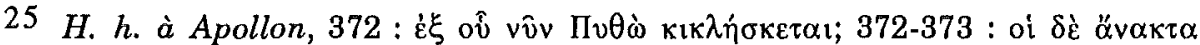

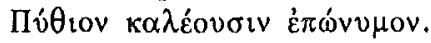


fait observer que $\pi \dot{v} \theta \varepsilon \sigma \theta \alpha \imath$ s'employait jadis dans le même sens que $\sigma \eta ́ n \varepsilon \sigma \theta \alpha \imath$ et invoque à ce sujet un passage célèbre de l'Odyssée (XII, 48) ${ }^{26}$. Pausanias a retenu cette interprétation qui évoquait le triomphe du $\operatorname{dieu}^{27}$ sans faire allusion à une autre "étymologie» qui mettait le nom de Pythô en rapport avec une forme de $\pi v v \theta \alpha ́ v \varepsilon \sigma \theta \alpha \iota^{28}$.

Une des traditions les plus curieuses concerne la ville de Lykôreia. Nous avons vu qu'au début de son exposé, Pausanias avait fait intervenir Parnassos, qui fonde la ville la plus ancienne, donne son nom au Parnasse et au val de Parnassia. On lui attribuait aussi l'invention de l'ornithomancie ${ }^{29}$. S'il faut en croire une autre version, il aurait été le premier à prophétiser à Pythô ${ }^{30}$. Il ne sera plus question ensuite de Parnassos. La ville qu'il avait fondée est engloutie par le déluge au temps de Deucalion $(\mathrm{X}, 6,2)$. Pausanias va maintenant nous conter les origines de Lykôreia.

Comme je l'ai rappelé précédemment ${ }^{31}$, le nom de Lykôreia trouverait son explication dans une étymologie qui associe le nom du loup

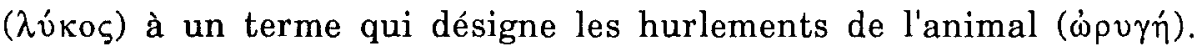
Cette étymologie fantaisiste ne manque pas d'ingéniosité et elle s'adapte aux circonstances. Il y avait des loups dans le massif du Parnasse. Quant au rôle de guides que la tradition confie aux loups du

26 C'est l'épisode des Sirènes, avec les chairs de leurs victimes qui "pourrissent" ( $\pi v \theta$ of́́vov). Sur ce texte, voir A. MoTTE, Prairies et jardins de la Grèce antique, Bruxelles, 1973, p. 159.

27 Sur la valeur religieuse de l'explication donnée dans la Suite Pythique, voir J. Defradas, Les thèmes de la propagande delphique, Paris, 1954, p. 64.

28 «Étymologie populaire» selon P. CHANTRAINE, Dictionnaire étymologique de la langue grecque, s.v. $\Pi v \theta \dot{\omega}$, notion qui dans un cas de ce genre me paraît discutable. SOPHOCLE avait déjà tiré parti du rapprochement avec $\pi v v \theta \alpha ́ v o \mu \alpha t$ : Edipe-Roi, 71 ( $\left.\pi \dot{\theta} \theta o v \theta^{\prime}\right), 604$ ( $\pi \varepsilon v \theta_{0}$ ); voir aussi STRABon, IX, 419 (avec une remarque sur la quantité de l'v dans la syllabe initiale); PLUT., De E Delphico, 2 (Mor., 385b), où sont expliquées plusieurs épiclèses du dieu. PaUsanias, X, 18, 2 cite un oracle avec la forme $\pi \varepsilon v \sigma o ́ \mu \varepsilon v o r$ (J. FonTENRose, The Delphic Oracle, Univ. of California, 1981, p. 315, Q 203). Les deux explications du nom de Pythô

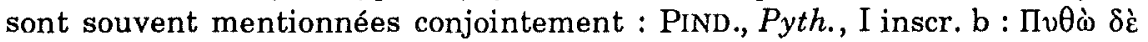

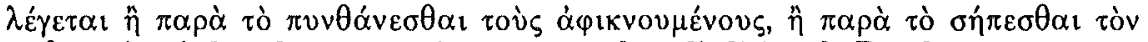
$\Pi v \theta \hat{\omega} \vee \alpha$ Év $\alpha$ v่n̂, et les textes cités en note dans l'édition de Drachmann.

29 P. AMANDRY, Mantique, p. 57, n LXIV; sur l'observation du vol des oiseaux, voir G. Roux, Delphes, p. 81-82.

30 Alexandre Polyhistor, 273 F 83 Jacoby (StéPH. Byz., s.v. П $\alpha \rho v \alpha \sigma \sigma o ́ \varsigma$ ); P. AMANDRY, Mantique, p. 57, $\mathrm{n}^{\circ}$ LXXXI (attribué à AleXANDRIDÈs).

31 Voir ci-dessus, p. 267. 
Parnasse $e^{32}$, il trouve son équivalent dans de nombreuses légendes où un animal sert d'intermédiaire à la divinité pour découvrir un objet, localiser le site d'une ville ou l'emplacement d'un oracle. À Delphes, le fameux loup de bronze offert par les Delphiens commémorait, disait-on, l'intervention d'un loup qui avait permis de récupérer "l'or sacré", volé dans le sanctuaire et caché dans le Parnasse ${ }^{33}$. L'oracle lui-même, selon une légende bien connue, aurait été découvert grâce à un troupeau de chèvres ${ }^{34}$.

P. Amandry a rappelé récemment que l'emplacement de Lykôreia reste une énigme, sans que l'on puisse toutefois prétendre qu'il s'agit d'une ville légendaire ${ }^{35}$. Par ailleurs, on peut affirmer que Lykôreia est étroitement associée à Delphes. Pour Strabon ${ }^{36}$, c'était un ancien établissement des Delphiens, situé sur la hauteur, au-dessus du sanctuaire. Selon le scholiaste d'Apollonios de Rhodes ${ }^{37}$, les Delphiens étaient appelés jadis $\Lambda \cup \kappa \omega \rho \varepsilon i \varsigma s$, du nom d'une bourgade $(\kappa \omega ́ \mu \eta)$, Lykôreia. O. Jahn a fait observer depuis longtemps ${ }^{38}$ que Lykôreia a été tirée de son obscurité par le goût des poètes alexandrins pour le rare et l'étrange. Elle était citée par Callimaque ${ }^{39} . \Lambda v \kappa \omega \rho \varepsilon v ́ \varsigma$ et $\Lambda v \kappa \omega ́ p \varepsilon\llcorner o \varsigma$ sont

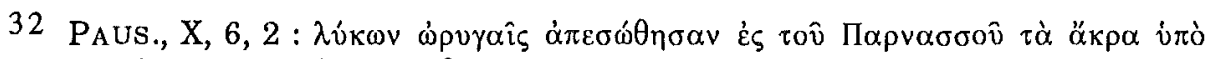

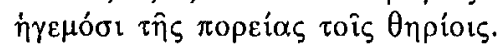

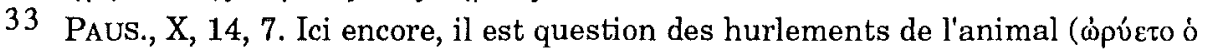
$\lambda$ úko̧). Sur cette offrande, voir W. RICHTER, $R E$, Suppl. XV (1978), s.v. Wolf, col. 976; sur son emplacement, voir G. DAux, Pausanias à Delphes, p. 157.

34 Textes cités par P. AmANDry, Mantique, p. 50, n. 2, avec le texte de Diodore, XVI, 26, cité et traduit, p. 51, n XXVIII; voir aussi G. Roux, Delphes, p. 65.

35 P. AMANDRY, L'Antre Corycien, in BCH, Suppl. VII (1981), p. 32, n. 4.

36 Après sa célèbre description du site de Delphes, comparé à un théâtre,

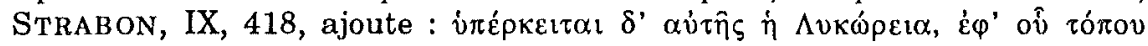

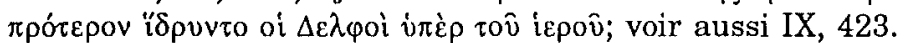

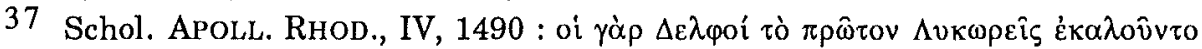

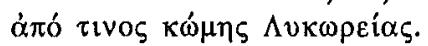

38 O. JAHN, Über Lykoreus, in Berichte über die Verhandlungen der sächs. Ges. der Wiss. zu Leipzig, I (1846-1847), p. 418 : «Die Vorliebe der alexandrinischen Dichter für das Seltene und Entlegene zog das dunkle Lykoreia ans Licht, welches als ein gewählter Name für Delphi gebraucht wurde»; voir aussi Bölte, RE, XIII (1927), s.v. Lykoreia, col. 2383. Krisa et Kirrha ont aussi servi à désigner Delphes; voir L. LERAT, Krisa (cité n. 10), p. 627 : «c'est devenu une élégance d'employer en poésie Krisa et Kirrha au lieu de Delphes». Sur la préférence accordée par les poètes aux anciens noms, voir à propos de Dymé, qui se serait appelée jadis Paleia, la remarque de PAUSANIAS, VII, 17, 7.

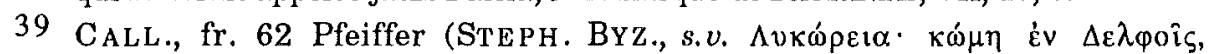

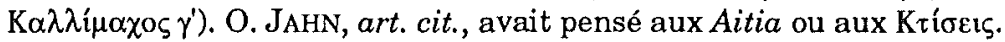


des épithètes d'Apollon chez les poètes de l'époque hellénistique et grécoromain $\mathrm{e}^{40}$. Le terme $\Lambda v \kappa \omega \rho i \tau \alpha \imath$ apparaît chez Pausanias ${ }^{41}$ pour désigner une population qui résidait dans la région du Parnasse. Il est certain que la tradition relative à la fondation de Lykôreia avec le récit du déluge et l'histoire des loups qui, par leurs hurlements, guident les rescapés jusqu'aux sommets du Parnasse ne remonte pas à une époque ancienne $\mathrm{e}^{42}$. Elle porte la marque d'une érudition qui a connu un grand développement aux temps hellénistiques et dans les siècles suivants ${ }^{43}$.

La mention de Deucalion à propos des origines de Lykôreia ( $\kappa \alpha \tau \grave{\alpha}$ $\Delta \varepsilon v \kappa \alpha \lambda i \omega v \alpha ; X, 6,2)$ pose, me semble-t-il, un problème. Deucalion n'était pas un inconnu à Delphes : on en faisait le père d'Amphictyon, l'éponyme de l'Amphictyonie ( $\mathrm{X}, 8,1)$, et un excellent témoin, Plutarque, nous apprend que le collège des Hosioi l'avait choisi comme ancêtre ${ }^{44}$. Une tradition ancienne, déjà connue de Pindare ${ }^{45}$, localisait sur le Parnasse le débarquement de Deucalion et Pyrrha. Ils avaient trouvé refuge dans un coffre $(\lambda \alpha \dot{\alpha} \rho \alpha \xi)$ et, selon certains érudits, le terme $\lambda \alpha \rho \vee \alpha \xi$ serait à l'origine du nom du Parnasse, appelé jadis "Larnassos» 46

Là où on attendait l'histoire du déluge dans sa version tradition-

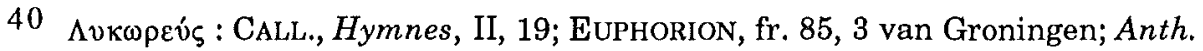

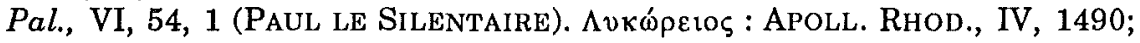
appliqué à la cithare du dieu : Anth. Plan., 279, 4.

41 Paus., IV, 34, 9, à propos de l'origine des habitants d'Asiné (Messénie), qui

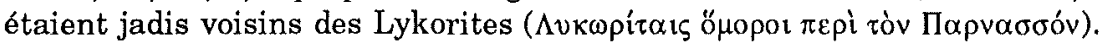

42 Voir BöLTe, RE, XIII (1927), s.v. Lykoreia, col. 2382 : «Der späte Ursprung dieser Erzählung verrät sich in der künstlichen Etymologien; voir aussi J. SCHMIDT, RE, XVIII, 2 (1949), s.v. Parnassos, col. 1633.

43 Selon BöLTE, art. cit., un poète alexandrin pourrait être à l'origine de cette tradition; voir aussi J. SCHMIDT, art. cit., qui met la légende en rapport avec l'histoire contée par HÉRodote, VIII, 32 (cf. PAUS., X, 32, 9).

44 Plut., Quest. gr., 9 (Mor., 292d); P. AmANDRY, Mantique, p. 123, nº XXXVII; sur le collège des Hosioi, voir G. Roux, Delphes, p. 59 sq.

45 PIND., Ol., X, 43. La légende figurait déjà dans les Catalogues hésiodiques et dans les Généalogies d'Acousilaos : HÉs., fr. 2 Merkelbach-West (schol. APOLL. RHOD., III, 1086); ACousilados, 2 F 35 Jacoby (schol. PIND., Ol., IX, 70a).

46 ANDRON D'HALICARNASSE expliquait comment, par un changement de lettre, Larnassos avait pu devenir Parnassos : 10 F 8 Jacoby (schol. APOLL. RHOD., II, 711 ; Etym. Magn., 655, 5); sur cette forme de la légende, voir les textes cités par J. ScHMIDT, RE, XVIII, 2 (1949), s.v. Parnassos, col. 1633. Voir aussi H. UsENER, Die Sintfluthsagen, Bonn, 1899, p. 40; TÜmPEL, RE, V (1905), s.v. Deukalion, col. 267. Sur le rôle du coffre, voir E. BRüMMER, Griech. Truhenbehälter, in JDAI, 100 (1985), p. 119-120. 
nelle, telle que nous la connaissons par les manuels de mythologie ${ }^{47}$, Pausanias nous offre une variante qui, tout en situant le déluge dans la région du Parnasse, se distingue par son originalité. Peut-être a-t-il cédé à ce goût du rare et de l'étrange qu'O. Jahn avait décelé chez les poètes de l'école alexandrine. Il nous a en tout cas conservé un bel exemple d'une érudition qui s'attache aux noms de lieux, aux interprétations étymologiques que l'on peut en donner et aux légendes que l'on peut tirer de ces interprétations.

Un autre exemple, non moins significatif, mérite d'être rappelé, car on y retrouve les mêmes données et le même procédé dans l'élaboration de la légende. Il s'agit cette fois de Mégaros, l'éponyme de Mégare, et de la manière dont il échappa au déluge : les cris des grues ( guidèrent jusqu'aux sommets d'une montagne qui reçut pour cette raison le nom de Gérania (I, 40,1). Cette tradition d'origine mégarien$n \mathrm{e}^{48}$ ressemble singulièrement à la tradition delphique; elle est construite sur une interprétation étymologique où les grues du mont Gérania jouent un rôle analogue aux loups de Lykôreia.

$J$ 'en reviens aux éponymes, dont il a été question précédemment, et parmi lesquels on doit sans doute ranger Hyamos, fils de Lykôros. On serait tenté de mettre son nom en rapport avec celui d'Hyampeia, une des Phédriades, et on resterait ainsi dans le paysage delphique. Mais le scholiaste d'Euripide fait d'Hyamos l'éponyme de Hyampolis, ville phocidienne déjà citée dans le Catalogue des vaisseaux (Il., IV, 521)49.

47 Ps.-APOLL., Bibl., I, 46-48 Wagner. C'est la version qui doit sa célébrité aux Métamorphoses d'OVIDE, I, 313 sq. Selon une autre tradition, conservée par l'auteur de la chronique de Paros (239, A ep. 2 et 4 Jacoby), Deucalion, après avoir régné à Lykôreia, se serait enfui au moment du déluge pour se réfugier à Athènes, où il fonda le sanctuaire de Zeus Olympien. Sur l'origine attique de cette tradition, voir E. DoPP, Quaestiones de Marmore Pario, diss. Rostok, 1883, p. 6; LAqUeUR, RE, XIV (1930) s.v. Marmor Parium, col. 1889. Sur la chronologie qui faisait remonter ces événements à une haute époque, voir L. ROBERT, $B C H, 102$ (1978), p. 473.

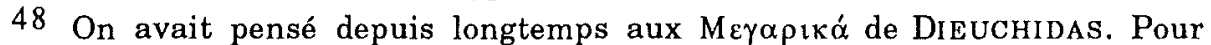
K. Hanel, Megarische Studien, 1934, p. 82, il s'agirait plutôt d'un poète de l'époque hellénistique, mais voir le commentaire de JACOBY, $485 \mathrm{~F} 1$ et $487 \mathrm{~F} 4$. Autre explication du nom de Géraneia qui serait dû, selon le scholiaste de THuCYDide, I, 105, 3, à la forme de la montagne.

49 Schol. Eur., Or., 1094, avec une généalogie dont BöLTE, RE, LX (1916), s.v. Hyampolis, col. 21, a noté le caractère arbitraire («willkürlich»). Selon la tradition que nous a conservée PAUSANIAS, X, 35, 5, Hyampolis, appelée d'abord

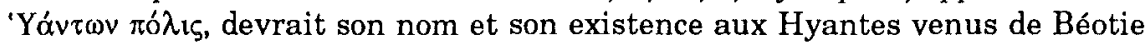


Il existe toutefois un autre problème qui mérite davantage de retenir notre attention. Les généalogies de Delphos attribuent un rôle important à Mélaina; elle apparaît dans ces généalogies sous diverses formes : Mélainis, Mélanthô, Mélantheia, Kélainôo ${ }^{50}$. On n'a pas hésité à mettre Mélaina en rapport avec Gé $\left(\gamma \alpha \hat{\imath} \alpha \mu \varepsilon^{\prime} \lambda \alpha \iota v \alpha\right)$ ou encore à l'associer au culte de Dionysos ${ }^{51}$. Aux spéculations des modernes mythographes on doit préférer, me semble-t-il, les réalités géographiques. Un texte de Dioscoride, que nous a conservé Galien ${ }^{52}$, nous apprend qu'il existait dans la plaine de Krisa ${ }^{53}$, au pied du Kirphis, un bourg appelé Mélainai

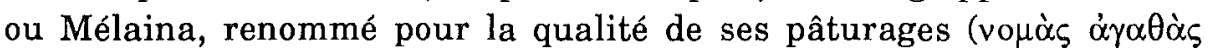

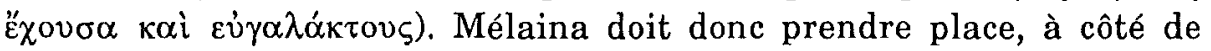
Parnassos, de Kôrykia et de Kastalios dans la liste des éponymes qui évoquent certains aspects du paysage delphique ${ }^{54}$. Elle est le produit d'une érudition qui aimait à reconstituer un passé légendaire à l'aide de traditions locales. Si l'on cherche des éléments de comparaison, on en trouvera en Attique avec le dème de Mélainai et son éponyme Mélanthos ${ }^{55}$, en Arcadie avec Mélaineai, fondée, disait-on, par Mélaineus, fils de Lykaon ${ }^{56}$.

50 Sur ces généalogies, voir ci-dessus, n. 19.

51 Dans un ouvrage réédité récemment (Python, Univ. of California Press, 1980), J. FONTENROSE écrit (p. 47) : «Melantho may be a name of Ge, since the Greeks often called the earth melaina (black)». Il invoque les généalogies et conclut : «Therefore Melantho or Melaina is a consort of Poseidon, who is often the mate of Ge or Demetern. Utiliser de cette manière les combinaisons élaborées par les érudits anciens me paraît un exercice périlleux. L'interprétation qui voulait faire de Delphos, fils de Melaina, un homme de race noire est tout aussi fantaisiste; voir là-dessus mes Etudes d'archéologie numismatique, p. 40 et l'article Delphos d'Erika SIMON in LIMC.

52 Galien, Lexique d'Hippocrate, XIX, p. 120 Kühn; voir aussi [HIPPOCRATE], Ep., 27 (IX, p. 406 Littré).

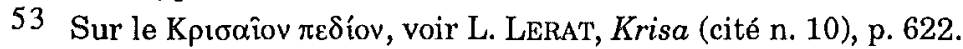

54 U. von Wilamowitz, Pindaros, Berlin, 1922, p. 71-72 : «Die Krisaeer hatten manche Dörfer, darunter Melaina an der Kirphis, nach dem die Mutter des Delphos heisst”.

55 Sur Mélanthos, voir J. ToepfFer, Attische Genealogie, Berlin, 1889, p. 231; U. voN WILAMOWITZ, Aristoteles und Athen, II (1893), p. 129. Sur le rôle de ce héros dans la légende étiologique des Apaturies, voir M.P NiLsson, Griech. Feste, 1906 (réimpression 1957), p. 464; W. KROLL, RE, XV (1932), s.v. Melanthos, col. 435.

56 PAus., VIII, 26, 8; cf. M. JosT, Sanctuaires et cultes d'Arcadie, Paris, 1985, p. 74. Autres Mélaina ou Mélainai en Troade, en Cilicie et en Lydie : L. RoBErT, Noms indigènes dans l'Asie Mineure gréco-romaine, Paris, 1963, p. 407. 
G. Daux a fait observer que «la périégèse delphique est encombrée de toutes sortes de $\lambda$ ó ${ }^{\prime}$, historiques, géographiques, mythologiques, cynégétiques» 57 . L'observation s'applique tout particulièrement aux commentaires qui accompagnent la description de certaines offrandes 58 , mais ces commentaires répondent à un souci d'explication qui est partout présent dans l'œuvre de notre auteur. L'introduction à la périégèse delphique, comme nous avons pu le constater, est elle-même un assemblage de logoi, empruntés à diverses sources et appartenant à des époques diverses. Ces logoi ont permis à Pausanias de retracer l'histoire de Delphes aux temps les plus anciens, qui sont nécessairement des temps mythiques, précédant et rejoignant les temps historiques ${ }^{59}$. La renommée de l'oracle assurait à Delphes un patrimoine légendaire particulièrement vénérable et qui n'a cessé de s'enrichir. Pausanias nous en a conservé une partie. On notera le rôle attribué aux noms de lieux et aux légendes qui se sont créées au sujet de leur origine. Tantôt on a eu recours à un éponyme, tantôt à une «étymologie». Les deux types d'explication peuvent du reste coexister et le lecteur de Pausanias est amené à les confronter. Il arrive que le Périégète se contente de les juxtaposer ou encore qu'il prenne parti pour l'une d'entre elles ${ }^{60}$. Ces observations ne me paraissent pas dépourvues

57 G. Daux, Pausanias à Delphes, p. 2.

58 Nous en avons vu un exemple avec l'explication que l'on donnait du loup de bronze (ci-dessus $n$. 33). On en trouverait un autre avec une offrande des Tarentins (PAUS., X, 13, 10), où la présence d'un dauphin, emblème de la cité, avait conduit à imaginer la légende du dauphin qui aurait sauvé Phalanthos d'un naufrage. Sur cette question, voir mon livre Monnaies et colonisation dans l'Occident grec, Bruxelles, 1965, p. 90. Parmi les numismates, il en est encore qui continuent à appeler Phalanthos le cavalier au dauphin des monnaies de Tarente.

59 Dans sa dissertation sur la chronique de Paros (citée ci-dessus n. 47), E. DopP écrivait (p. 10) : "Historici graeci, priusquam ad suae aetatis res enarrandas accederent, imprimis id egerunt, ut tempora, ut ita dicam, mythica cum historicis aliquo modo coniungerent». Sur cette façon d'écrire l'histoire, voir mon article in Bull. Acad. royale de Belgique, Classe des Lettres (1980), p. 193 sq.

60 Les deux explications citées pour le second temple d'Apollon (PAUS., X, 5, 10)

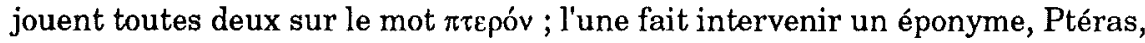

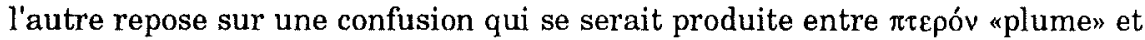
$\pi \tau \varepsilon$ p $\zeta$ *fougère». PAUSANIAS condamne ce second logos. Autre exemple avec le nom de l'Amphictyonie (PAUS., X, 8, 1) qui s'expliquerait par un éponyme, Amphictyon, ou par une étymologie. On peut rapprocher de cette seconde explication, qui avait pour elle l'autorité de l'atthidographe ANDROTION (324 F 58 Jacoby), celle qui est admise par les savants modernes : P. Chantraine, 
d'intérêt. Elles se limitent à vrai dire aux origines mythiques de Delphes. Mais elles nous aident à mieux connaître la pensée et les préoccupations d'un homme qui n'est pas seulement un guide pour l'archéologue. Il est aussi le témoin d'un passé légendaire dont la survie lui doit beaucoup 61 .

Rue des Glacis, 153

Léon LACROIX

B - 4000 LIÈGE

Dictionnaire étymologique, s. $v$. кті́ $\omega$. On notera que le héros Amphictyon avait un sanctuaire dans un des sièges de l'Amphictyonie, aux Thermopyles (HDT. VII, 200), autre exemple d'un éponyme que l'on ne peut ne considérer comme une création tardive.

61 W. GuRlitr, Über Pausanias, Graz, 1890, p. 139 sq., avait bien souligné l'importance du patrimoine légendaire, constituant un véritable folklore, que l'on pouvait encore recueillir à l'époque de PAUSANIAS dans les différentes régions de la Grèce. Il avait rappelé aussi (p. 144) le rôle joué dans la survie de ce patrimoine par les poètes dits alexandrins. 